Article

\title{
The Influence of Photoelectron Escape in Radiation Damage Simulations of Protein Micro-Crystallography
}

\author{
Hugh Marman, Connie Darmanin * and Brian Abbey * \\ ARC Centre of Excellence in Advanced Molecular Imaging, Department of Chemistry and Physics, \\ La Trobe Institute for Molecular Science, La Trobe University, Melbourne, Victoria 3086, Australia; \\ hjmarman@students.latrobe.edu.au \\ * Correspondence: c.darmanin@latrobe.edu.au (C.D.); b.abbey@latrobe.edu.au (B.A.); \\ Tel.: +613-9479-5367 (C.D.); +613-9479-2645 (B.A.)
}

Received: 1 May 2018; Accepted: 24 June 2018; Published: 27 June 2018

\begin{abstract}
Radiation damage represents a fundamental limit in the determination of protein structures via macromolecular crystallography (MX) at third-generation synchrotron sources. Over the past decade, improvements in both source and detector technology have led to MX experiments being performed with smaller and smaller crystals (on the order of a few microns), often using microfocus beams. Under these conditions, photoelectrons (PEs), the primary agents of radiation-damage in MX, may escape the diffraction volume prior to depositing all of their energy. The impact of PE escape is more significant at higher beam energies $(>20 \mathrm{keV})$ as the electron inelastic mean free path (IMFP) is longer, allowing the electrons to deposit their energy over a larger area, extending further from their point of origin. Software such as RADDOSE-3D has been used extensively to predict the dose (energy absorbed per unit mass) that a crystal will absorb under a given set of experimental parameters and is an important component in planning a successful MX experiment. At the time this study was undertaken, dose predictions made using RADDOSE-3D were spatially-resolved, but did not yet account for the propagation of PEs through the diffraction volume. Hence, in the case of microfocus crystallography, it is anticipated that deviations may occur between the predicted and actual dose absorbed due to the influence of PEs. To explore this effect, we conducted a series of simulations of the dose absorbed by micron-sized crystals during microfocus MX experiments. Our simulations spanned beam and crystal sizes ranging from $1 \mu \mathrm{m}$ to $5 \mu \mathrm{m}$ for beam energies between $9 \mathrm{keV}$ and $30 \mathrm{keV}$. Our simulations were spatially and temporarily resolved and accounted for the escape of PEs from the diffraction volume. The spatially-resolved dose maps produced by these simulations were used to predict the rate of intensity loss in a Bragg spot, a key metric for tracking global radiation damage. Our results were compared to predictions obtained using a recent version of RADDOSE-3D that did not account for PE escape; the predicted crystal lifetimes are shown to differ significantly for the smallest crystals and for high-energy beams, when PE escape is included in the simulations.
\end{abstract}

Keywords: macromolecular crystallography; radiation damage; photoelectron escape; Monte Carlo simulation; nanocrystals

\section{Introduction}

Technological advances over the last few decades have helped to mitigate, though not eliminate, radiation damage in protein crystallography. They have also facilitated the use of smaller, less perfect crystals in structure determination. Experiments can be performed using microfocus $X$-ray beams with sufficient flux density to cause rapid degradation of crystals. The use of such highly-focused beams is 
set to become routine in the near future, as diffraction-limited storage rings, currently in construction or planning at several facilities worldwide, begin to come on-line [1,2]. The characteristics of small crystals, combined with these developments, create new challenges for understanding the effects of radiation damage. For an overview of the field, the reader is directed toward the recent reviews by Garman [3-6] and Holton [7].

Several metrics have been proposed to track the progression of radiation damage. These include loss of integrated intensity in the diffraction patterns, increase in scaling B-factors, expansion of the unit-cell, reduction of metal centres, and loss of resolution in susceptible residues [3]. These metrics are measured against the dose (in MGy) received by a crystal, being the energy absorbed per unit mass. Dose is not measured directly but is calculated based on the relevant beam and sample parameters.

The total diffracted intensity is one of the simplest metrics used for tracking X-ray induced damage to macromolecules [7]. The integrated intensity of the complete diffraction volume, a specific wedge of diffraction data, or the intensity at a single Bragg peak, have all been used to study radiation damage [3]. The integrated intensity of an individual Bragg spot can be modelled with the empirical formula

$$
I(t)=I_{0} \exp \left(-\ln (2) \frac{D(t)}{H d}\right)
$$

where $I$ and $D$ are the spot intensity and dose received at time $t$, respectively, $I_{0}$ is the intensity at $t=0$, $d$ is the resolution of the Bragg spot, and $H=10 \mathrm{MGy}^{-1}$ is the Howell's criterion [8]. Howell's criterion was derived using spot fading to half the initial intensity as an indication of dose limit [7]. As higher resolution spots fade faster, Equation (1) therefore provides a resolution-dependent estimate of the dose that a crystal can absorb before the diffracted intensity falls to half its initial level. Holton has shown that Equation (1) shows good agreement with recent damage studies [7].

Dose prediction is, therefore, a critical factor when planning an MX experiment, especially if the amount of crystalline sample is limited and/or the available crystals are limited in size. RADDOSE [9-12] is a software package that has been demonstrated to produce realistic dose estimates for MX experiments under typical conditions (e.g., crystal and beam sizes $>5 \mu \mathrm{m}$ and X-ray energies around $12 \mathrm{keV}$ ). Improvements in source and detector technology, however, mean that increasingly MX experiments are being carried out using micron-sized crystals and beams.

At the beam energies typically used in MX, X-ray-matter interactions are dominated by photoionisation, with incoherent scatter becoming appreciable at energies above $20 \mathrm{keV}$. During photoionisation, a photoelectron (PE) is ejected from a core shell (principally the K-shell since this has the largest cross-section) by an incident X-ray photon. The primary PE has a kinetic energy equal to the energy of the incident $X$-ray photon minus the core shell binding energy. The ejected $\mathrm{PE}$ undergoes deceleration via a series of inelastic collisions with the atomic electrons within the solid. The average distance travelled by the PE between these collisions is its inelastic mean free path (IMFP). Provided the energy of the absorbed X-ray photon is large enough and the size of the X-ray beam small enough, the PE may escape the diffracting volume of the sample prior to depositing all of its energy, leading to a reduction in radiation damage, relative to diffracted intensity. The beam size, rather than the crystal size, is the critical element here: if the beam is much larger than the crystal, approximately the same number of PEs will enter the crystal from the solvent as will escape it, offsetting the benefit due to PEs escape. Although the basic principles behind this damage reduction have been established the circumstances under which these effects may become important in MX experiments are still a topic of active research [13-18]. At the time this study was undertaken, the most widely used programmes for determining dose in MX experiments did not yet account for the possibility of PE escape, a fact that has only recently changed with the release of a new version of RADDOSE-3D [19]. Neglecting the possibility of PE escape is expected to result in a lower-than-predicted dose rate to the crystal, provided the beam and crystal size are small enough and the X-ray energy high enough $[20,21]$. Establishing the conditions under which PE escape is expected to have a significant impact on dose rates for micron-sized crystals is a key aim of the current work. 
The last decade has seen a growing body of literature exploring the potential benefits of PE escape for MX through both theoretical models and experiments. These studies have explored the length scales and energy ranges over which a reduction in radiation damage might be realised due to PE escape, providing some practical solutions for minimising radiation damage [20,21]. Suggestions generally rely on making the beam footprint smaller (in at least one dimension) than the damage footprint. This may be accomplished by using microfocus or line-focus X-ray beams [16-18]. Some special cases have also been investigated, where PE escape might be enhanced, these include imaging of platelet or needle-like crystals [22]. Previous studies $[17,20,21]$ of the effect of PE escape have often incorporated Monte Carlo (MC) modelling of electron trajectories, performed using programmes such as CASINO [23,24]. In the current paper, we present the first systematic simulation study of PE escape in MX accounting for:

(1) the effects of a finite beam size comparable to the PE stopping range;

(2) the effects of a finite crystal size matched to the FWHM of the beam;

(3) the effect of variation in X-ray beam energy; and

(4) the influence of the beam profile as a function of the dose received by the crystal over time.

In contrast to previous work, we explicitly simulated the distribution of dose due to both PE escape and Compton scattering in the whole beam footprint, accounting for PEs produced in the crystal and the surrounding solvent. These spatially-resolved simulations of dose distribution were used to update the time-resolved damage state of the crystal. By comparing spatially-resolved dose maps produced with and without PE motion incorporated, we arrived at an estimate of the experimental conditions under which the effects of PE escape could lead to a substantial reduction in radiation damage.

\section{Results}

We simulated the dose absorbed by a lysozyme crystal that was fixed in place and did not rotate. We used 2D (cross-sectional) dose maps produced according to Section 4.5, and 3D dose maps produced using RADDOSE-3D [9], as inputs for a spot-fading model (Section 4.7) that simulated the loss in integrated intensity of a $3 \AA$ Bragg spot under a range of beam- and sample-conditions. In this section, we present:

- the results of our spot-fading model, comparing the effects of variation in crystal size, beam size, and beam energy;

- calculations of the change in X-ray interaction cross-sections under our simulation parameters, and the implications this has for diffraction efficiency in micro-crystallography; and

- a representation of a $2 \mathrm{D}$ slice through the real-space diffraction volume, illustrating how the contribution to diffracted intensity within this volume evolves as the crystal absorbs a dose of up to 60 MGy.

We note that RADDOSE-3D produces several aggregate dose metrics; when comparing global dose rates, we used the average dose, whole crystal (ADWC).

\subsection{Crystal Size}

Figure 1 shows plots of the ratio of integrated intensity to initial intensity, $I / I_{0}$ (Equation (1)), as a function of time for cubic crystals with edge lengths of $1 \mu \mathrm{m}, 1.5 \mu \mathrm{m}, 2 \mu \mathrm{m}$ and $5 \mu \mathrm{m}$. Simulations based on three sets of dose maps are compared. We produced one set of dose maps that accounted for PE escape and a second (control) set that did not; both included the effect of Compton scattering. The dose maps produced using RADDOSE-3D did not include either PE escape or Compton scattering. In all cases, the beam energy was fixed at $12 \mathrm{keV}$ with a beam flux of $1 \times 10^{10}$ photons s$^{-1}$; the beam FWHM was matched to the side length of the crystal. The influence of PE escape on the rate of spot-fading was found to be significant for the $1 \mu \mathrm{m}$ crystal, with simulations that account for PE escape predicting that the peak intensity falls to half its initial value in approximately $40 \mathrm{~s}$, four times longer than predictions that do not account for PE escape. The discrepancy between simulations with and without PE escape 
was found to decrease steadily as the crystal/beam size increased up to $5 \mu \mathrm{m}$, beyond which no significant difference was seen between the two approaches. No significant difference was observed, for any crystal size, between the two simulations that did not account for PE escape.

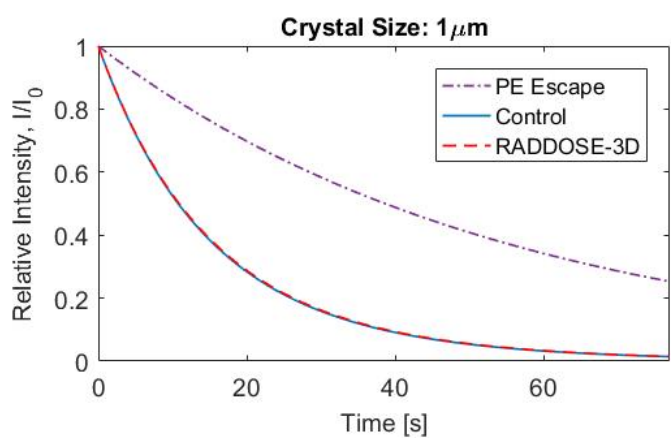

(a)

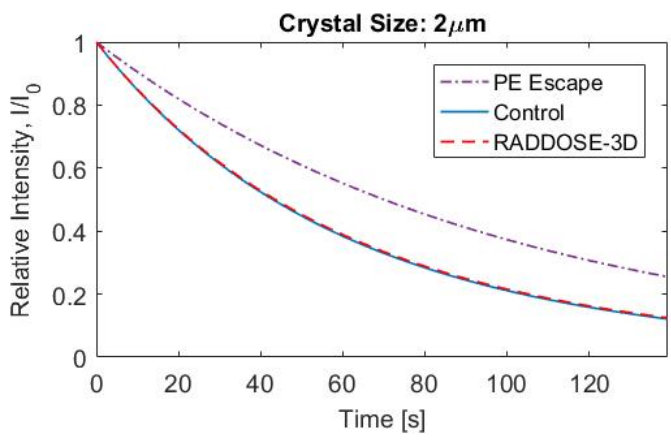

(c)

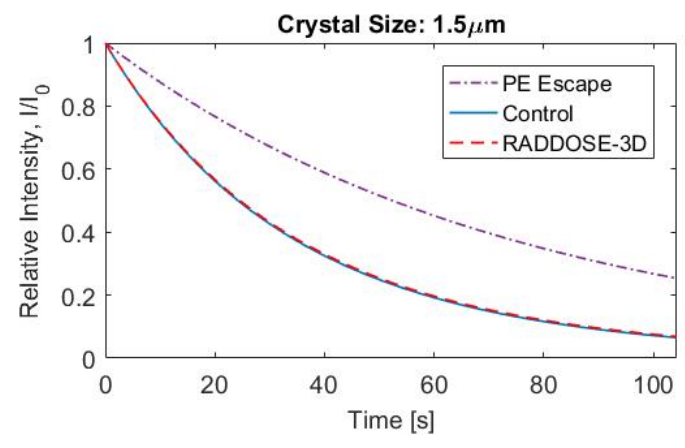

(b)

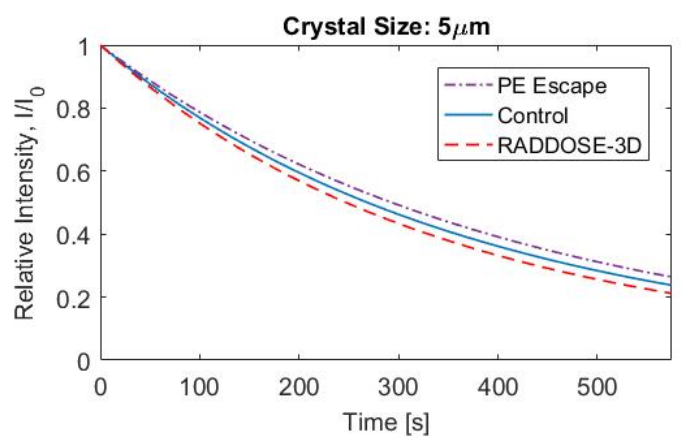

(d)

Figure 1. Relative intensity, $I / I_{0}$, as a function of time, for a $3 \AA$ Bragg spot for crystal size: (a) $1 \mu$; (b) $1.5 \mu \mathrm{m}$; (c) $2 \mu \mathrm{m}$; and (d) $5 \mu \mathrm{m}$. Each crystal is assumed to be centred in a $12 \mathrm{keV}$ beam with incident flux of $1 \times 10^{10}$ photons s ${ }^{-1}$ and a beam FWHM matched to the crystal size. Three sets of dose maps were used in the simulations: Purple dot-dash lines and solid blue lines show results based on our simulated dose maps (see Section 4.5). The former accounted for PE escape, the latter (control) did not; both accounted for Compton scattering. Our model that accounted for PE escape also included the effect of PEs being scattered into the crystal from the solvent. Dashed red lines show results based on RADDOSE-3D [9] dose maps; the version used did not account for either PE escape or Compton scattering. The discrepancy between the simulation that accounts for PE escape and those that do not becomes more significant as the crystal size decreases from $5 \mu \mathrm{m}$ to $1 \mu \mathrm{m}$. No significant variation is observed between the two simulations that did not account for PE escape.

\subsection{Beam Size}

Figure 2 shows plots of the relative integrated intensity, $I / I_{0}$, as a function of time, with the crystal side-length fixed at $1 \mu \mathrm{m}$ and the beam FWHM varying from $1 \mu \mathrm{m}$ to $5 \mu \mathrm{m}$. The beam energy and incident flux were fixed at $12 \mathrm{keV}$ and $1 \times 10^{10}$ photons s$^{-1}$, respectively. As expected, when the beam FWHM was matched to the $1 \mu \mathrm{m}$ crystal size, there was a significant discrepancy between the simulations that included PE escape and those that did not. This discrepancy reduced as the beam FWHM was increased up to $5 \mu \mathrm{m}$, at which point it was no longer significant. No significant variation was seen between the two simulations that did not include PE escape, for any beam size. 


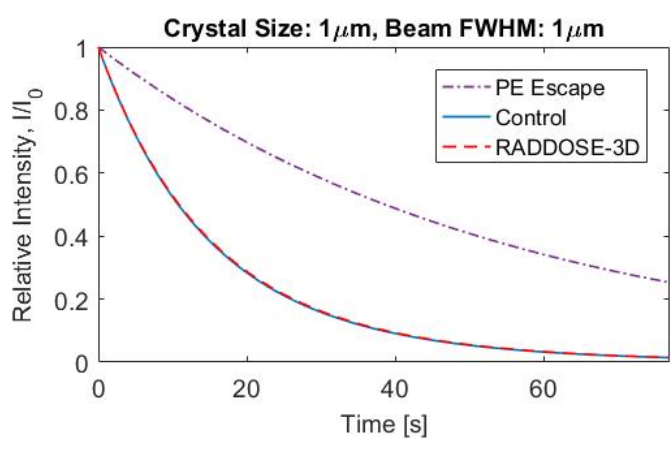

(a)

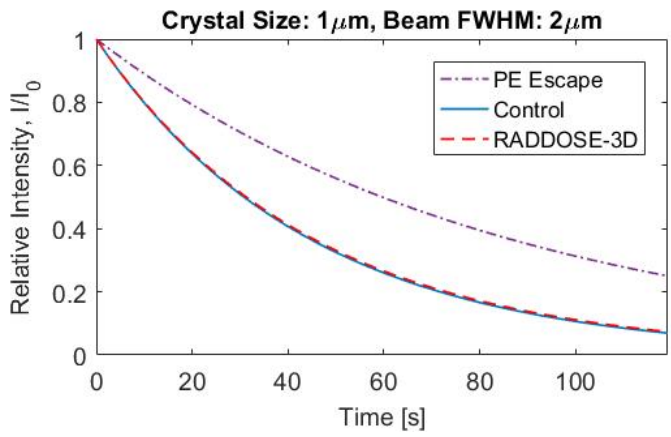

(c)

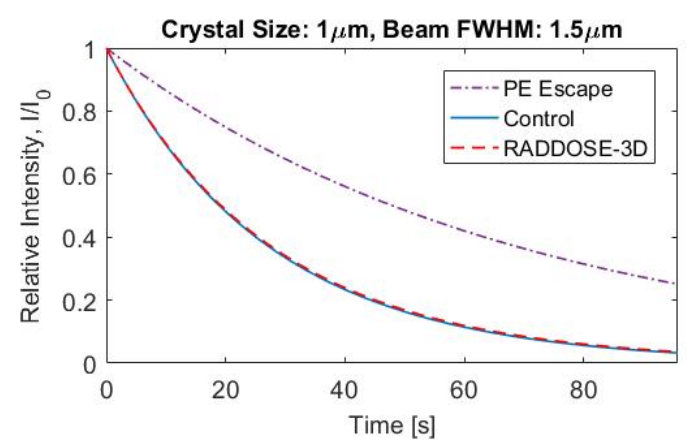

(b)

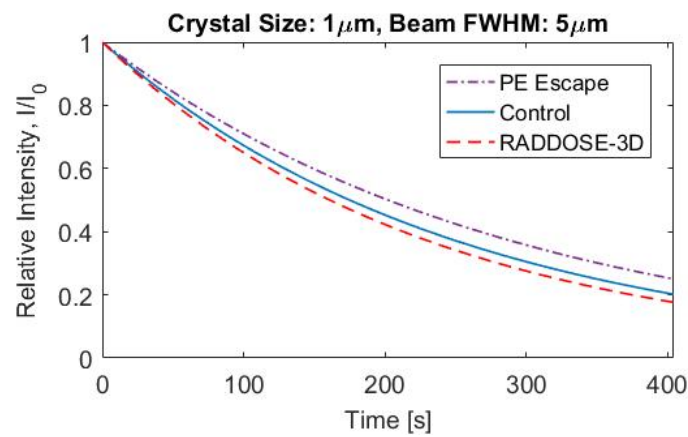

(d)

Figure 2. Relative intensity, $I / I_{0}$, as a function of time, for a $3 \AA$ Bragg spot generated by a $1 \mu \mathrm{m}$ crystal centred in X-ray beams of varying width. All beams had energy of $12 \mathrm{keV}$ and incident flux of $1 \times 10^{10}$ photons s $^{-1}$. The beam FWHM was: (a) $1 \mu \mathrm{m}$; (b) $1.5 \mu \mathrm{m}$; (c) $2 \mu \mathrm{m}$; and (d) $5 \mu \mathrm{m}$. Three sets of dose maps were used in the simulations: Purple dot-dash lines and solid blue lines show results based on our simulated dose maps (see Section 4.5). The former accounted for PE escape, the latter (control) did not; both accounted for Compton scattering. Dashed red lines show results based on RADDOSE-3D [9] dose maps; the version used did not account for either PE escape or Compton scattering. The discrepancy between the simulation that accounts for PE escape and those that do not becomes more significant as the beam size decreases from $5 \mu \mathrm{m}$ to $1 \mu \mathrm{m}$. No significant variation is observed between the two simulations that did not account of PE escape.

\subsection{Beam Energy}

Figure 3 shows plots of the variation in $I / I_{0}$ as a function of time for a range of different beam energies $(9 \mathrm{keV}, 12 \mathrm{keV}, 15 \mathrm{keV}, 20 \mathrm{keV}, 25 \mathrm{keV}$ and $30 \mathrm{keV})$. The beam FWHM was fixed at $1 \mu \mathrm{m}$, matched to the $1 \mu \mathrm{m}$ crystal size. For all energies simulated, slower spot fading was predicted when PE escape was included in the model. The difference between the two different simulations was small at $9 \mathrm{keV}$, but increased steadily up to $20 \mathrm{keV}$ for the simulated lysozyme crystal. Beyond $20 \mathrm{keV}$, the discrepancy continued to increase but at a much reduced rate. Between $9 \mathrm{keV}$ and $20 \mathrm{keV}$, no significant variation was seen between the two simulations that did not include PE escape. A small difference between the two was observed at $25 \mathrm{keV}$ and $30 \mathrm{keV}$, with our control (that did account for Compton scattering) predicting slightly faster spot-fading than RADDOSE-3D (which did not account for Compton scattering). 


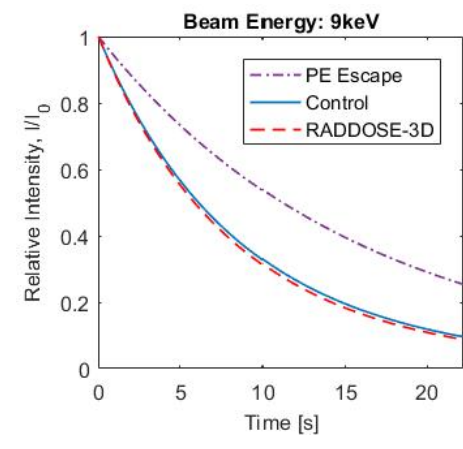

(a)

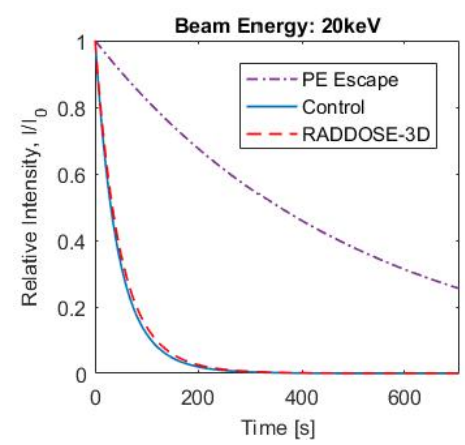

(d)

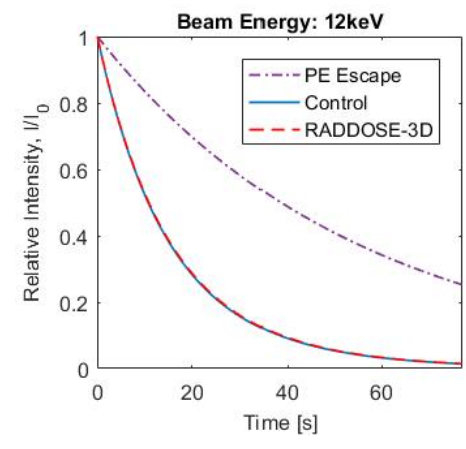

(b)

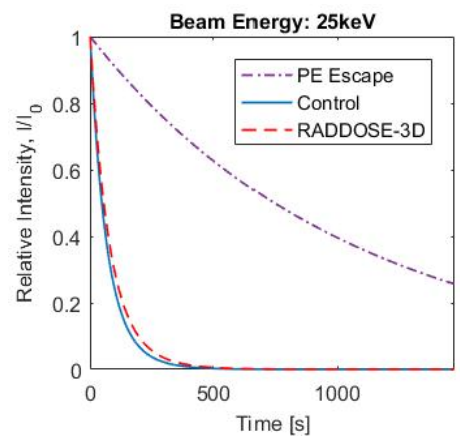

(e)

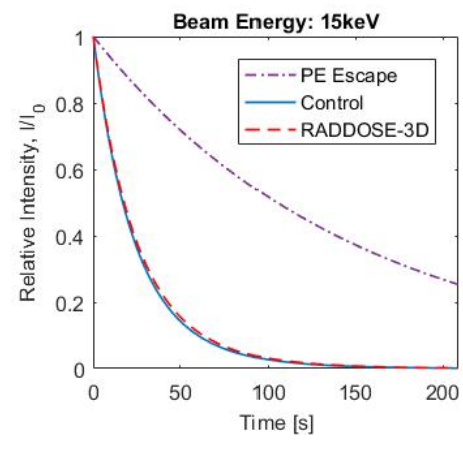

(c)

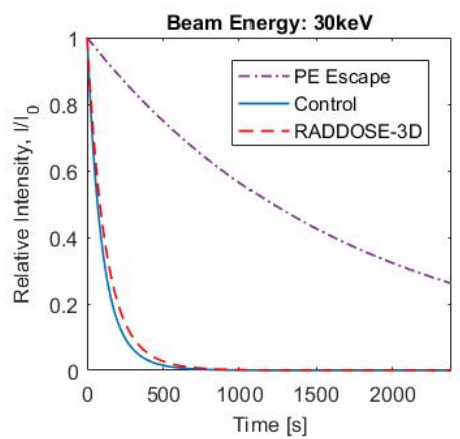

(f)

Figure 3. Relative intensity, $I / I_{0}$, as a function of time, for a $3 \AA$ Bragg spot generated by a $1 \mu \mathrm{m}$ crystal centred in a $1 \mu \mathrm{m}$ beam with an incident flux of $1 \times 10^{10}$ photons s$^{-1}$ and beam energy of: (a) $9 \mathrm{keV}$; (b) $12 \mathrm{keV}$; (c) $15 \mathrm{keV}$; (d) $20 \mathrm{keV}$; (e) $25 \mathrm{keV}$; and (f) $30 \mathrm{keV}$. Three sets of dose maps were used in the simulations: Purple dot-dash lines and solid blue lines show results based on our simulated dose maps (see Section 4.5). The former accounted for PE escape, the latter (control) did not; both accounted for Compton scattering. Dashed red lines show results based on RADDOSE-3D [9] dose maps; the version used did not account for either PE escape or Compton scattering. Incorporating PE escape resulted in lower damage rates being predicted at all energies, with increasing discrepancy seen between results as the energy increased from $9 \mathrm{keV}$ to $20 \mathrm{keV}$, and minimal change in discrepancy observed above $20 \mathrm{keV}$. Inclusion of Compton scattering did not result in any significant discrepancy between the two simulations that ignored PE escape for energies up to $20 \mathrm{keV}$ and resulted in only a small deviation at $25 \mathrm{keV}$ and $30 \mathrm{keV}$.

\subsection{Variation of Diffraction Efficiency with Energy}

We estimated the diffraction efficiency, $D E\left(E, \sigma_{X}, \sigma_{B}\right)$, of our simulated lysozyme crystals (see Section 4.1 for sample details) as a function of crystal size, $\sigma_{X}$, beam size, $\sigma_{B}$, and beam energy, $E$, by two methods that are described in Section 4.8. Results are grouped into three categories, which are summarised in Table 1:

(1) $D E_{N I S T}$ values were calculated using cross-section data for coherent scatter, $\sigma_{C}$, incoherent scatter, $\sigma_{I}$, and photoelectric absorption, $\sigma_{P}$, obtained from the NIST XCOM database [25]. These results did not account for PE escape and were independent of beam and crystal size.

(2) $D E_{N o P E}$ values were calculated as the ratio of the number of coherently scattered photons per unit volume, $N_{C} / \sigma_{X}^{3}$, to the average global dose, $D_{G}$, based on results of our simulations that did not account for PE escape-this included both our control simulations and simulations that used RADDOSE-3D dose maps. $D E_{N o P E}$ was insensitive to changes in beam and crystal size; within each set (RADDOSE-3D, control) results at $30 \mathrm{keV}$ showed a standard deviation of $3 \%$ from the average, with averages at all other energies showing a standard deviation of less than 
$1 \%$. Results from our control and RADDOSE were within 1-2\% of each other at energies up to $15 \mathrm{keV}$ but diverged at higher energies, differing by $4 \%$ to $10 \%$ between $20 \mathrm{keV}$ and $30 \mathrm{keV}$. A full table of $D E_{N o P E}$ values is shown in Appendix A; average values were used in Table 1 and average values with error bars showing the standard deviation were used in Figure 4.

(3) $D E_{P E}$ values were calculated the same way as $D E_{N o P E}$ values but using the results of the simulations that did include PE escape. They showed significant dependence on beam size and some dependence on crystal size for a given beam size. $D E_{P E}$ values for a $1 \mu \mathrm{m}$ crystal in a $1 \mu \mathrm{m}$ beam are shown in Table 1. A plot of all $D E_{P E}$ values is shown in Figure 4.

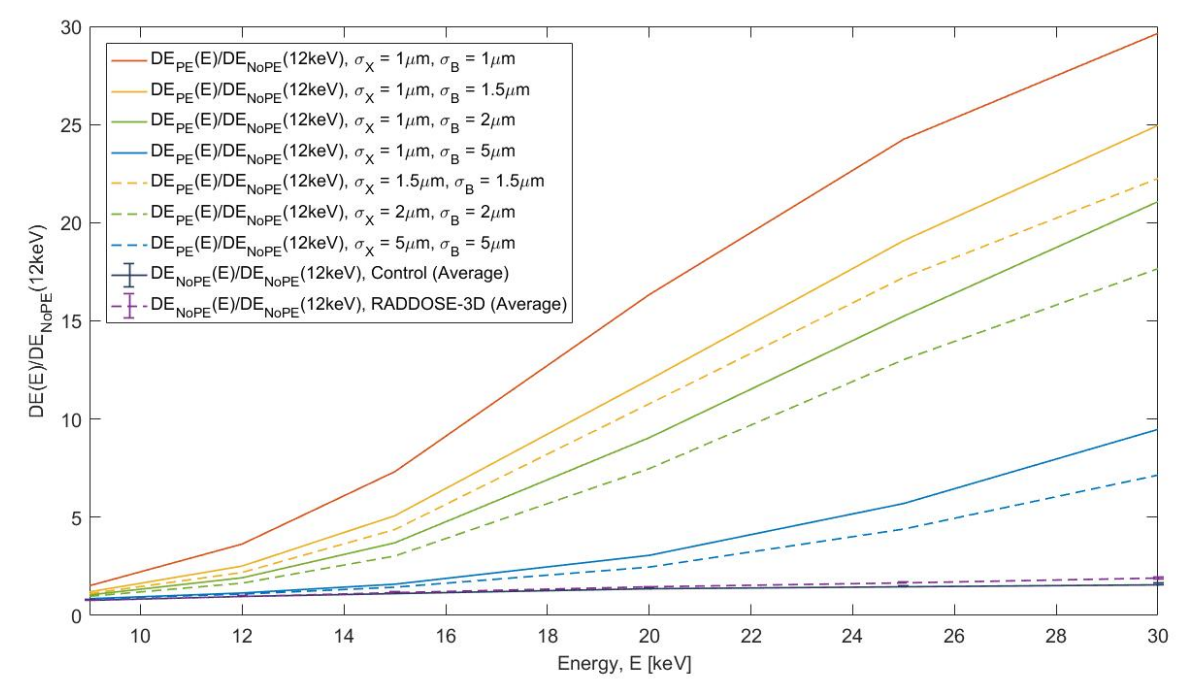

Figure 4. Diffraction efficiency, $D E(E)$, of simulated results with PE escape ( $\left.D E_{P E}\right)$ and without PE escape ( $\left.D E_{N o P E}\right)$ incorporated. Red, orange, green, and blue lines (both solid and dashed) are for $D E_{P E}$, crystals and beams between $1 \mu \mathrm{m}$ and $5 \mu \mathrm{m}$, respectively, as shown in the legend. The solid dark blue line is the average of $D E_{N o P E}$, as determined by our control simulations, across all crystal and beam sizes, with error bars showing standard deviation. The dashed purple line shows the average for RADDOSE-3D results. As the standard deviation within each set is $\leqslant 3 \%$ for all energies, the error bars are only resolvable on some data points. The reference value was the value of $D E_{N o P E}(12 \mathrm{keV})$ calculated with RADDOSE-3D; all values of $D E_{P E}(E)$ and $D E_{N o P E}(E)$ are relative to this reference.

As shown in Table 1, values of $D E_{N o P E}$ (based on both RADDOSE-3D and our controls) differed from those of $D E_{N I S T}$, for a given energy, by between $15 \%$ and $35 \%$. Both $D E_{N I S T}$ and $D E_{N o P E}$ were found to be essentially independent of beam and crystal size, and showed an approximate doubling between $12 \mathrm{keV}$ and $30 \mathrm{keV}$, although this factor was lower for our control simulations. As shown in Figure 4, values of $D E_{P E}$ were only similar to those of $D E_{N o P E}$ for the $1 \mu \mathrm{m}$ and $5 \mu \mathrm{m}$ crystals in the $5 \mu \mathrm{m}$ beam at energies $\leqslant 15 \mathrm{keV}$. Across all the simulated results that included PE escape, the ratio $D E_{P E}\left(E, \sigma_{X}, \sigma_{B}\right) / D E_{N o P E}(12 \mathrm{keV})$ varied between $1.09\left(\sigma_{X}=\sigma_{B}=5 \mu \mathrm{m}\right)$ and $3.63\left(\sigma_{X}=\sigma_{B}=\right.$ $1 \mu \mathrm{m})$ at $E=12 \mathrm{keV}$, with the greatest difference occurring at $30 \mathrm{keV}\left(7.15\left(\sigma_{X}=\sigma_{B}=5 \mu \mathrm{m}\right)\right.$ and $\left.29.64\left(\sigma_{X}=\sigma_{B}=1 \mu \mathrm{m}\right)\right)$. 
Table 1. Cross-section data and diffraction efficiency for a lysozyme crystal with $36 \%$ solvent, as described in Section 4.1, relative to the reference values $D E_{N I S T}(12 \mathrm{keV})$ and the RADDOSE-3D [9] value for $D E_{N o P E}(12 \mathrm{keV})$. Cross-sections for coherent scatter, $\sigma_{C}$, incoherent scatter, $\sigma_{I}$, and photoelectric absorption, $\sigma_{P}$, were calculated using the NIST XCOM database [25]. Diffraction efficiency was calculated as the ratio $\sigma_{C} /\left(\sigma_{I}+\sigma_{P}\right)$ from cross-section data and the ratio of coherently scattered photons per unit volume, $N_{C} / \sigma_{X}^{3}$, to average global dose, $D_{G}$, for simulations. $D E_{N o P E}$ values are averages across all beam and crystal sizes simulated. $D E_{P E}$ values are for a $1 \mu \mathrm{m}$ crystal in a $1 \mu \mathrm{m}$ beam.

\begin{tabular}{rcccccc}
\hline Energy, $\boldsymbol{E},[\mathbf{k e V}]:$ & $\mathbf{9}$ & $\mathbf{1 2}$ & $\mathbf{1 5}$ & $\mathbf{2 0}$ & $\mathbf{2 5}$ & $\mathbf{3 0}$ \\
$\sigma_{C}:$ & 1.44 & 1.00 & 0.74 & 0.49 & 0.35 & 0.26 \\
$\sigma_{I}+\sigma_{P}:$ & 2.35 & 1.00 & 0.53 & 0.25 & 0.15 & 0.11 \\
\hline$D E_{N I S T}(E) / D E_{N I S T}(12 \mathrm{keV}):$ & 0.61 & 1.00 & 1.41 & 1.98 & 2.29 & 2.32 \\
\hline$D E_{N o P E}(E) / D E_{N o P E}(12 \mathrm{keV}), \mathrm{Control}:$ & 0.81 & 0.99 & 1.15 & 1.37 & 1.51 & 1.59 \\
$D E_{N o P E}(E) / D E_{N o P E}(12 \mathrm{keV}), \mathrm{RADDOSE}-3 \mathrm{D}:$ & 0.79 & 1.00 & 1.19 & 1.47 & 1.69 & 1.91 \\
\hline$D E_{P E}(E) / D E_{N o P E}(12 \mathrm{keV}):$ & 1.51 & 3.63 & 7.32 & 16.33 & 24.25 & 29.64 \\
\hline
\end{tabular}

\subsection{Evolution of Diffraction Volume as a Function of Dose}

Figure 5 shows a series of 2D slices through the diffraction volume of a $1 \mu \mathrm{m}$ crystal centred in a $12 \mathrm{keV}, 1 \mu \mathrm{m}$ X-ray beam for global doses ranging from $0 \mathrm{MGy}$ to $60 \mathrm{MGy}$. We observe that at lower doses the shape of the diffraction volume is dominated by the Gaussian profile of the beam. As the crystal continues to absorb radiation, the diffraction volume changes in several ways: the overall volume shrinks; the strength of the diffracted signal (both peak and average) drops; and the shape of the volume becomes broader, an effect that is stronger along the axis perpendicular to the polarisation direction of the X-ray beam.

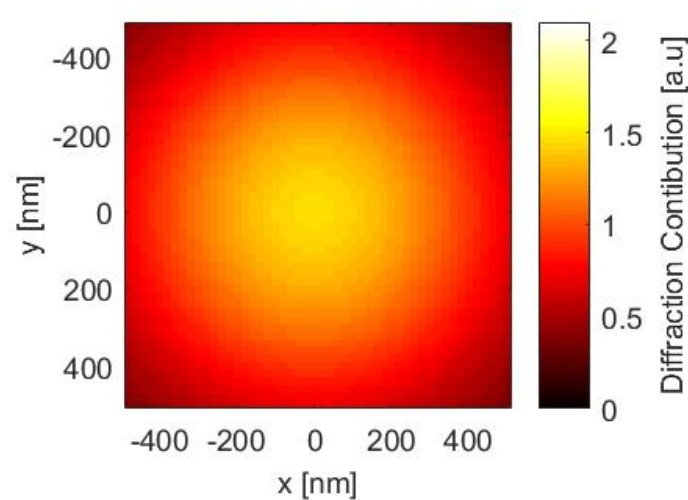

(a)

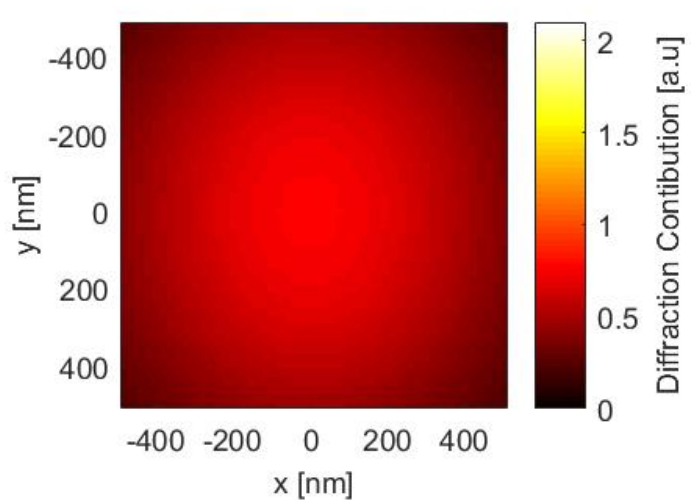

(b)

Figure 5. Cont. 


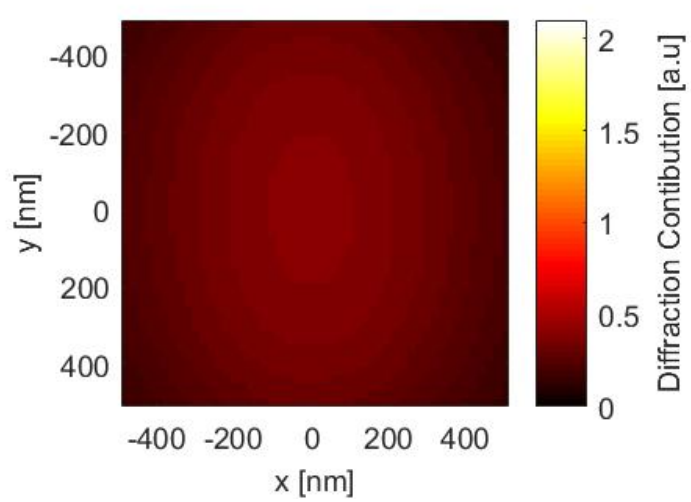

(b)

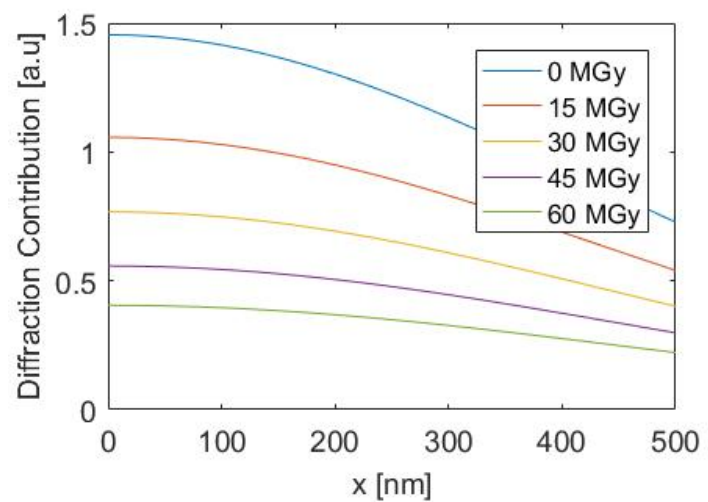

(c)

Figure 5. Heat map showing diffraction volume, as a function of global dose, for a $1 \mu \mathrm{m}$ crystal centred in a $12 \mathrm{keV}, 1 \mu \mathrm{m}$ beam (FWHM). The heat maps show the contribution to diffracted signal of a $2 \mathrm{D}$ slice perpendicular to the beam direction, when the crystal has absorbed a global dose of: (a) 0 MGy; (b) 30 MGy; and (c) 60 MGy. Panel (d) summarises the drop in diffraction volume along the $x$-axis (parallel to the polarisation axis of the $\mathrm{X}$-ray beam), including additional calculations after the crystal has absorbed doses of $15 \mathrm{MGy}$ and $45 \mathrm{MGy}$.

\section{Discussion}

A multi-stage approach was used to explicitly model the average distribution of energy in a crystal (and the surrounding solvent) due to both photoelectric absorption and Compton scattering. Simulations that accounted for PE escape produced predictions of damage progression in MX that agreed with results of our control simulation and RADDOSE-3D (both of which neglected PE escape) when the crystal and beam sizes were greater than $5 \mu \mathrm{m}$ and the beam energy was less than $12 \mathrm{keV}$, but diverged when both the crystal and beam size were reduced below $5 \mu \mathrm{m}$ and/or the beam energy was increased. As discussed below, this divergence in results is consistent with existing models of damage-reduction via PE escape. Moreover, our approach permits a qualitative examination of the progression of crystal deterioration in real-space, a research topic that is the subject of intense investigation, both theoretically [26] and experimentally [14,15].

For simplicity, we did not include rotational effects in our simulations. For many of the configurations we simulated, rotation would result in a more even spread of dose across the crystal volume (particularly at the edges) but the key effect being explored (the impact of PE escape on damage reduction) would not be significantly affected by rotation of the crystal.

\subsection{Prediction of Extended Lifetimes for Small Crystals and Beams, and Higher Energies}

Our findings suggest that in some cases the lack of inclusion of PE escape leads to deviations from the behaviour predicted by RADDOSE-3D [9] (see, for example, [14,16,17]). This conclusion is supported by both the similarity in output to RADDOSE-3D under conditions where PE escape is unlikely to affect dose (or when it has been ignored in our models), and the divergence in output under conditions where PE escape is expected to be significant.

A comparison of Figures 1 and 2 shows that results that account for PE escape and those that do not exhibit a similar convergence as the beam width is increased, whether or not the crystal size is also increased so that it matches the beam width. The reason for this is that PEs are produced everywhere within the beam footprint, in both crystal and solvent. If the crystal is large, the majority of the PEs produced within it will not escape it. By contrast, if the crystal is small, many PEs will escape but if the beam footprint includes a significant portion of the surrounding solvent, a roughly equal number of PEs will be scattered into the crystal, offsetting the effect of those that escape it. For the sample that 
we modelled, the chemical compositions and densities of the crystal and surrounding solvent are not significantly different in terms of the production and propagation of PEs. This is a consequence of the fact that the cross-sections and average binding energies between solvent and crystals are quite similar, leading to only a small difference in energy-deposition profile. Our choice of sample, lysozyme, contains no atoms heavier than sulphur and the only heavy atoms in the solvent we modelled are sodium and chlorine. The presence of more heavy atoms in either the crystal (e.g., metalloproteins) or the solvent (e.g., cacodylate) could lead to a more significant difference between the crystal and the solvent in the production of PEs.

The most striking discrepancy between results that included PE escape and those that did not occurred when simulating a $1 \mu \mathrm{m}$ crystal in a $1 \mu \mathrm{m}$ beam (FWHM) at high energies ( $\geqslant 15 \mathrm{keV}$ ) (Figure 3 ). Here, our simulations predict increased diffraction efficiency compared to RADDOSE-3D by a factor of close to $170 \%$ at $15 \mathrm{keV}, 300 \%$ at $20 \mathrm{keV}, 380 \%$ at $25 \mathrm{keV}$, and $400 \%$ at $30 \mathrm{keV}$. This discrepancy occurs because the IMFP of the PEs increases with energy and the resulting energy deposition is therefore spread over a larger area. The implication is that for micron-sized crystals illuminated by similarly sized beams, simulations that neglect PE escape over estimate the dose received, becoming increasingly less accurate as the incident $\mathrm{X}$-ray photon energy increases. It has been previously observed that conducting MX experiments with smaller crystals at higher energies may allow experimenters to maximise the reduced radiation damage afforded by PE escape $[17,20,21]$. However, a systematic experimental study, isolating the effect of beam-energy in radiation damage has not, to our knowledge, been carried out and will be the focus of further work.

\subsection{Simulations Enabling Qualitative and Semi-Quantitative Interpretation of Published Data}

In early simulation work carried out by Nave and Hill [21], they used simple CASINO models to estimate that an $8 \mu \mathrm{m}$ crystal illuminated with $30 \mathrm{keV}$ X-rays would benefit from a three-fold increase in diffraction efficiency due to PE escape. Although not included in their model, they noted the need to minimise the material surrounding the crystal to prevent PEs being scattered into it from the solvent, and the possibility of leveraging the anisotropic emission of PEs due to the beam polarisation to minimise damage. The largest crystal we simulated was $5 \mu \mathrm{m}$, which saw an approximate four-fold improvement in diffraction efficiency at $30 \mathrm{keV}$ when PE escape was accounted for; which is consistent with Nave and Hill's result. By including the solvent in our models, we have been able to gauge the effect of illuminating the volume around the crystal and, as discussed in the previous section, our results support the conclusion that PE production outside the crystal must be minimised if appreciable damage-reduction is to be achieved. Although Nave and Hill assumed larger beams and suggested physically limiting the volume of the solution surrounding the crystal, the increasing availability of microfocus X-ray beams may permit the same ends to be achieved through focusing, provided the crystal can be positioned within the beam with suitable precision to limit PE production outside the crystal.

Following, and extending upon, the work of Nave and Hill, Cowan and Nave [20] used coarse Monte Carlo models to study the impact of PE escape on diffraction efficiency. When neglecting PE escape, but accounting for Compton scattering, they observed a variation in diffraction efficiency of up to $40 \%$ between $1 \mathrm{keV}$ and $40 \mathrm{keV}$, with a plateau of maximum efficiency between $20 \mathrm{keV}$ and $30 \mathrm{keV}$. Under the same conditions, our model shows a similar trend, though the calculated values vary over a slightly wider range. Incorporating a model for PE escape, Cowan and Nave observed an improvement in diffraction efficiency at higher energies over a wide range of crystal sizes, though the effect was stronger for smaller crystals. In their model, a $1 \mu \mathrm{m}$ crystal saw a roughly five-fold increase in diffraction efficiency at $12 \mathrm{keV}$ when accounting for PE escape, with the improvement above $20 \mathrm{keV}$ being around 15-fold. This is consistent with our results (Figure 4) for the $1 \mu \mathrm{m}$ crystal in a $1 \mu \mathrm{m}$ beam, where the effect of PEs being generated in the solvent (which has been ignored in their model) is minimal. As in the model that excluded PE escape, Cowan and Nave's results show a broad plateau in diffraction efficiency for the $1 \mu \mathrm{m}$ crystal between $20 \mathrm{keV}$ and $30 \mathrm{keV}$, before it drops off slightly at 
higher energies. Our models also show a similar trend, though the diffraction efficiency (across all beam and crystal sizes) continues to improve as the energy is increased up to $30 \mathrm{keV}$, albeit at a reduced rate. This suggests that the increased cross-section for Compton scattering at higher energies offsets the reduction in dose due to PE escape. However, as shown in Figure 3, the discrepancy between our control simulation (that did included Compton scattering) and the RADDOSE-3D data (that did not include Compton scattering) is small, even at higher energies, and the discrepancy between them and the simulations that did include PE escape grows much more slowly above $20 \mathrm{keV}$ than it did between $9 \mathrm{keV}$ and $20 \mathrm{keV}$. Since this study was undertaken, RADDOSE-3D has been updated to include incoherent scatter and Bury et al. [19] observed that this inclusion only begins to have a significant effect on dose prediction above $40 \mathrm{keV}$. These results suggest that the increase in Compton scattering alone does not account for the plateau in diffraction efficiency at higher energies. A complementary interpretation is that much of the PE energy is already escaping the crystal at $20 \mathrm{keV}$ and the increase in electron IMFP above this energy simply serves to scatter the PEs further into the solvent. There is some support for this idea in Figure 4, as the gradient of the diffraction efficiency of the $1 \mu \mathrm{m}$ crystal in a $1 \mu \mathrm{m}$ beam (the configuration compared in Figure 3) drops off slightly at higher energies compared to the gradients for the larger crystals. Cowan and Nave [20], who simulated crystals up to $20 \mu \mathrm{m}$, observed a similar effect, with larger crystals continuing to benefit from increased diffraction efficiency as the beam energy was increased above the $20 \mathrm{keV}$ to $30 \mathrm{keV}$ where it plateaued for the $1 \mu \mathrm{m}$ crystal.

In Holton and Frankel's [27] paper on the minimum crystal size required to collect a complete data set, they estimated the effect of PE escape by first calculating the dose in the absence of PE escape and then scaling this dose by a so-called Nave-Hill capture factor, $f_{N H}$, which they approximated as

$$
f_{N H}=\frac{D_{P E}}{D_{N o P E}} \simeq \frac{R}{R+R_{P E}(E)}
$$

where $R$ was the radius of the spherical crystals they modelled, $R_{P E}$ was the range of a PE with energy $E$, and $D_{P E}$ and $D_{N o P E}$ were the dose values with and without accounting for PE escape, respectively. This capture factor was designed to equate to unity for "large" crystals at $12.4 \mathrm{keV}$ and was intended to provide only a coarse indication of the benefit of PE escape. We used global dose values from our simulations to calculate $f_{N H}$ as the ratio $D_{P E} / D_{N o P E}$. We also calculated $f_{N H}$ using the RHS of Equation (2), taking $R_{P E}$ as the maximum penetration depth of electrons in our CASINO simulations and substituting half the crystal edge-length for $R$. The two methods of calculation produced values for $f_{N H}$ within a factor of 2 of each other. Our simulations did not have the spherical-symmetry of those performed by Holton and Frankel, but, given the coarse nature of their estimate, this substitution is expected to provide a reasonable point of comparison. The similarity in values of $f_{N H}$ indicate that our simulation results are comparable to the Holton and Frankel model.

In addition to qualitative comparisons to previous modelling studies, a semi-quantitative assessment of our model can be conducted by comparison to recent results obtained by Finfrock et al. [16,18] and Sanishvili et al. [17], who performed experimental studies of radiation damage in large crystals by exposing sub-volumes using micro-focus and line-focus beams. They observed that the radiation damage footprint extended between $1.5 \mu \mathrm{m}$ and $5 \mu \mathrm{m}$ beyond the actual beam footprint, showing the spread of PEs beyond the diffraction volume. Both experimenters used beam energies close to $18 \mathrm{keV}$. In the Monte Carlo stage of our models (implemented using CASINO), energy-deposition extended approximately $5 \mu \mathrm{m}$ beyond the interaction point at $20 \mathrm{keV}$, which is in-line with these previous observations.

Since this study was conducted, a new version of RADDOSE-3D [19] has been released that includes Compton scattering and PE escape, neither of which was included in the version we used. As noted above, Bury et al. [19] found that the inclusion of Compton scattering made negligible difference below $20 \mathrm{keV}$, and only minimal difference between $20 \mathrm{keV}$ and $40 \mathrm{keV}$. Conversely, Bury et al. found the inclusion of PE escape to have a very significant impact on dose predictions for microcrystals. They simulated crystal sizes from $1 \mu \mathrm{m}$ to $100 \mu \mathrm{m}$ for a single beam energy of $12.4 \mathrm{keV}$ 
and observed a non-negligible reduction in dose for crystals as large as $20 \mu \mathrm{m}$ under these conditions. For a $10 \mu \mathrm{m}$ crystal, they observed a reduction in dose of approximately $20 \%$ when accounting for PE escape, and reduction of over $95 \%$ for a $1 \mu \mathrm{m}$ crystal. In our simulations, we observed a reduction of $72 \%$ for the $1 \mu \mathrm{m}$ crystal in a $12 \mathrm{keV}$ beam, when the beam FWHM was matched to the crystal (and a much lower reduction when the beam was larger). This discrepancy can be accounted for by the fact that we simulated the solvent around the crystal, thereby accounting for PEs scattered into the crystal as well as out of it, while the new version of RADDOSE-3D assumes an isolated crystal, neglecting the effect of the surrounding solvent. In the future, a comprehensive comparison of our simulations and this new version of RADDOSE-3D could provide useful insights.

\subsection{Permitting a Real Space Interpretation of Crystal Damage}

The idea that diffracted intensity is approximately proportional to crystal volume is well-established [7] and the implication is that damage leading to a reduced intensity of diffracted radiation can be interpreted in real-space as an apparent "shrinking" of the diffraction volume of the crystal. Note, this does not mean that any actual protein material is being lost from the volume of the initial crystal, but that it becomes so disordered that it no longer contributes meaningfully to the diffraction signal. Recently, Coughlan et al. $[14,15]$ reported such shrinking of the diffraction volume when using Bragg coherent diffractive imaging (BCDI) to produce 2D and 3D real-space images of lysozyme crystals that had been subjected to high dose burns (several hundred MGy).

Warkentin et al. [26] also recently modelled the change in the intensity of diffracted radiation per unit area for a crystal in a Gaussian beam, as it undergoes radiation damage. They observed that, due to the flux being highest in the beam centre, the portion of the crystal nearest the beam centre produced the largest number of diffracted photons, but also underwent radiation damage the fastest. Crucially, however, their model did not account for PE escape. To estimate which regions of the crystal contributed the most diffracted photons at the detector, they weighted each contribution by both the flux density in that part of the beam and the degree of damage already suffered. They observed a broadening of this contribution over time; initially, the central portion of the crystal contributed most strongly to the diffracted signal but by the time the spot intensity had dropped to half its initial value, their models predicted that the contribution to diffracted intensity was more or less equal across the whole FWHM of the beam. After sufficient exposure, radiation damage progressed to the point that only the fringes of the crystal (those in the lower flux edge of the Gaussian beam) contributed coherent scattered photons.

The snapshots of the diffraction volume in Figure 5 show a reduction in the total contribution to diffracted signal and shrinking and flattening of the diffraction contribution-meaning photons contributing to Bragg peaks are coming from different regions of the crystal. The flattening and shrinking of the diffraction volume is anisotropic as PEs are preferentially scattered at lower angles to the polarisation axis of the $\mathrm{X}$-ray beam (here the $x$-axis), resulting in slower damage rates along the axis perpendicular to polarisation. As highlighted in the summary graph in Figure 5, our simulations show the same trend as Warkentin's models [26], with an initial peak in the centre of the crystal and a flattening of the diffraction contribution across the whole crystal with increased dose. The overall shrinking of the diffraction volume is also in-line with the observations of Coughlan et al. $[14,15]$ and may provide an initial model for future experimental examination of this phenomenon.

\subsection{Micro-Crystallography Could Benefit Significantly from Higher X-Ray Energies}

Predictions produced by our model suggest significant benefits could be gained by optimising MX experiments along multiple parameter-axes, particularly when using smaller crystals and higher beam energies. The increase in electron IMFP at higher energies, coupled with a decrease in damage-causing interactions relative to elastic scatter, suggests that, all other things being equal, the greatest increase in diffraction efficiency could be gained from using higher energy beams. As highlighted in Table 1 , although the coherent scattering cross-section falls with increasing beam energy, it does so more slowly 
than the combined cross-sections for incoherent scattering and photoelectric absorption. Although experiments at higher energy take longer (roughly twice as long at $20 \mathrm{keV}$ and four times as long at $30 \mathrm{keV}$ ) due to the reduced cross-section for coherent scattering, the ratio of diffracted intensity to dose-accumulation (damage) would be roughly doubled for experiments above $20 \mathrm{keV}$, relative to those at $12 \mathrm{keV}$. This is even before accounting for the effect of PE escape; as shown in Figure 4, our simulations predict that $\mathrm{PE}$ escape could be responsible for increasing diffraction efficiency by up to 30 times, relative to the diffraction efficiency at $12 \mathrm{keV}$ in cases where PE escape is not considered. This is assuming the use of micro-crystals and micro-focus beams so that the effect of PE escape is large, but even for a $5 \mu \mathrm{m}$ crystal/beam, our calculations show PE escape contributing to an increase in diffraction efficiency. Given the relative rate at which the cross-sections change and the longer IMFP for higher-energy PEs, conducting experiments at higher energies may be advantageous even with larger crystals in more conventional MX experiments.

Several experiments [16-18] have shown reduced damage occurring due to PE escape, but have only explored a narrow parameter space thus far; a more thorough examination, particularly focussed on the benefit of using higher energy beams, could be guided by our results and data obtained could subsequently be used to validate and improve our model. Should experiments show quantitative agreement with our predictions, our model could be used to provide more accurate predictions of dose rate, and therefore crystal lifetime in micro-crystallography experiments, compared to currently available radiation damage software packages.

\section{Materials and Methods}

We simulated energy deposition within a lysozyme crystal and surrounding solvent (1:1 mixture of polyethylene glycol 400 (PEG400) and $0.5 \mathrm{M}$ acetic acid with $9 \% \mathrm{NaCl}(w / v)$ ). Crystal size was varied between $1 \mu \mathrm{m}$ and $5 \mu \mathrm{m}$. The incident beam was simulated with a fixed flux of $1 \times 10^{10}$ photons s$^{-1}$ at energies of $9 \mathrm{keV}, 12 \mathrm{keV}, 15 \mathrm{keV}, 20 \mathrm{keV}, 25 \mathrm{keV}$ and $30 \mathrm{keV}$, with FWHM from $1 \mu \mathrm{m}$ to $5 \mu \mathrm{m}$. These simulations produced 2D dose maps, showing slices through the diffraction volume perpendicular to the beam direction. By incorporating Monte Carlo modelling of electron trajectories (using CASINO) we produced dose maps that accounted for the motion of PEs and the spreading of their energy over an area surrounding their point of origin. These dose maps accounted for the generation of PEs everywhere in the beam footprint, including the solvent surrounding the crystal. We produced a control set of dose maps that did not simulate PE motion but included Compton scattering. We used a version of RADDOSE-3D [9] that did not include PE escape or Compton scattering to produce 3D dose maps with the same range of sample- and beam-parameters. All three sets of dose maps produced assumed that the crystal was not rotated and was centred in the beam. We subsequently used both 2D and 3D dose maps as inputs for time-resolved simulations that modelled the rate at which radiation damage accrued and the effect of this damage on the diffraction volume. We simulated the drop in integrated intensity of a $3 \AA$ Bragg spot, based on Equation (1). Variation in the diffraction volume of the crystal was modelled based on its spatially-resolved damage state and intensity of coherent scatter. A flow diagram of the simulations is shown in Figure 6; a sectioned explanation of the stages and input/output is given below; subheadings correspond to the labels of individual processes in the flow diagram. 


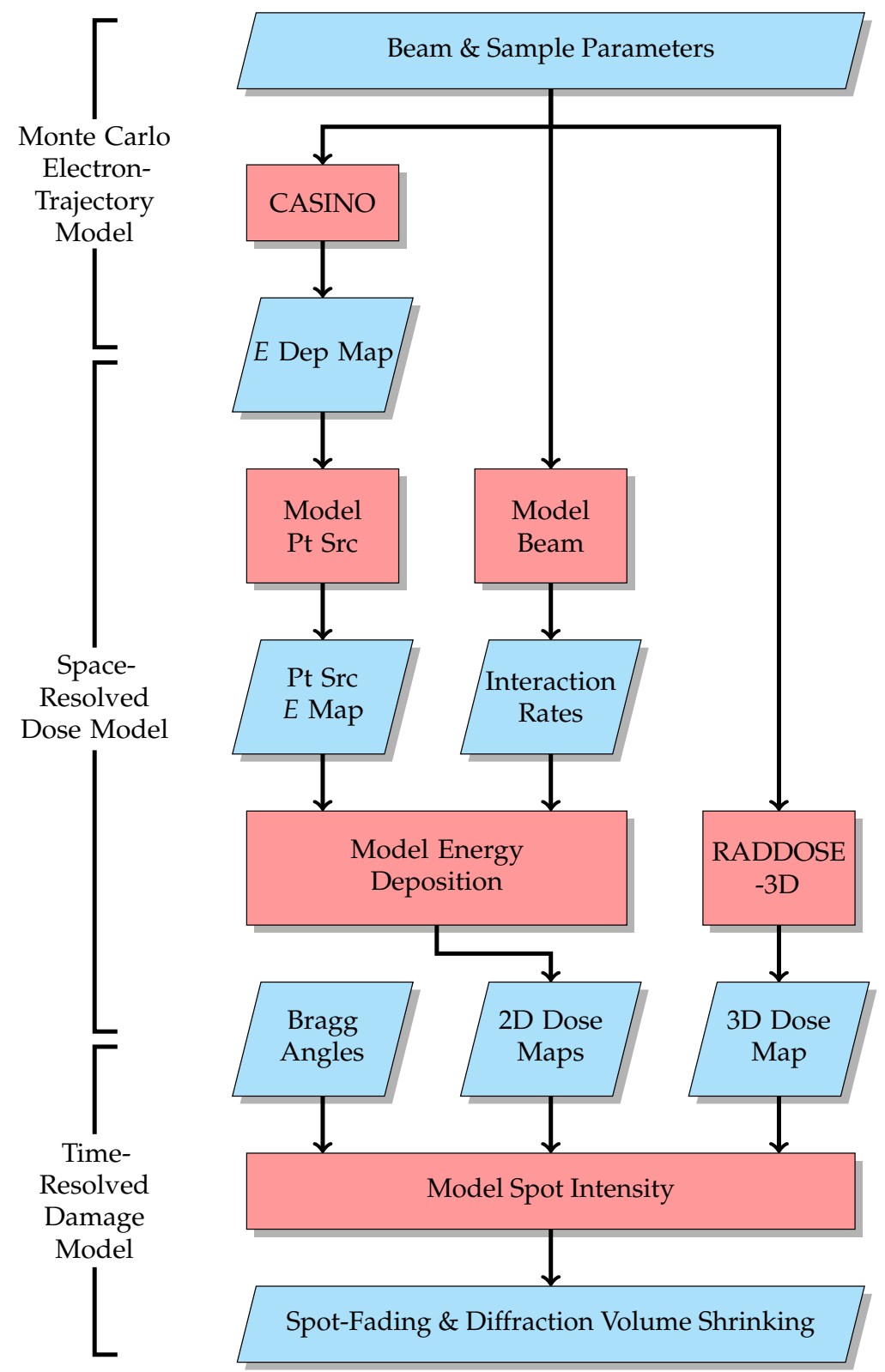

Figure 6. Flow diagram of our simulations, which were performed using a combination of 3rd-party applications, CASINO (red) and RADDOSE-3D [9] (pink), and our own MATLAB scripts (orange). Rectangles represent processes, parallelograms are inputs/outputs. CASINO output (E Dep Map) gives (ensemble-averaged) energy-deposition of an electron with a particular initial direction; post-processing of this data in MATLAB produces a dose map for a point source of photoelectrons with emission-direction weighted for a horizontally polarised X-ray beam. MATLAB scripts are further used to determine the dose footprint of the X-ray beam, accounting for spatially-distributed energy deposition by photoelectrons. RADDOSE-3D [9] is used to produce a dose map of the crystal that does not account for photoelectron escape. Our final MATLAB script models the loss in integrated intensity of a $3 \AA$ Bragg spot with and without accounting for photoelectron escape.

\subsection{Beam and Sample Parameters}

Our simulated sample was crystallised lysozyme, suspended in a 1:1 mixture of PEG400 and $0.5 \mathrm{M}$ acetic acid with $9 \% \mathrm{NaCl}(w / v)$, following crystallisation and sample preparation protocols by Coughlan [15]. The solvent content of the crystal was calculated to be $36 \%$. The sample parameters used in the simulation were chemical composition, density, and average ionisation potential (weighted by 
elemental cross-section and chemical composition). Crystals were modelled as cubes, with edge lengths of $1 \mu \mathrm{m}, 1.5 \mu \mathrm{m}, 2 \mu \mathrm{m}$ and $5 \mu \mathrm{m}$.

Different $X$-ray beam widths and energies were modelled; in each case, the total beam flux was $1 \times 10^{10}$ photons s $^{-1}$. X-ray beam widths modelled were $1 \mu \mathrm{m}, 1.5 \mu \mathrm{m}, 2 \mu \mathrm{m}$ and $5 \mu \mathrm{m}$. Energies modelled were $9 \mathrm{keV}, 12 \mathrm{keV}, 15 \mathrm{keV}, 20 \mathrm{keV}, 25 \mathrm{keV}$ and $30 \mathrm{keV}$. Cross-sections for photoionisation, Compton scattering, and elastic scattering were calculated using NIST XCOM online cross-section database [25].

\subsection{CASINO}

Monte Carlo simulations of electron trajectories were performed with CASINO (version 2.4.8.1) [23,24]. We simulated two different materials: the crystal, permeated by solvent, and the solvent alone. Both are described above in Section 4.1. For each material, we ran the simulation at six different (electron) energies. The energy in this case was the calculated average photoelectron energy, determined by the X-ray beam energy $(9 \mathrm{keV}, 12 \mathrm{keV}, 15 \mathrm{keV}, 20 \mathrm{keV}, 25 \mathrm{keV}$ and $30 \mathrm{keV})$ and the average photoionisation energy of the sample. CASINO is configured using up to five menus under the "Settings" tab; the settings we used are detailed below, under headings that correspond to the menus. Any parameters that are not explicitly stated below were left at their defaults. We made no adjustments from default values to either the "Runtime Options" or "Change Physical Models" menus.

\subsubsection{Modify Sample}

A single layer was used for each material. The thickness was varied depending on the beam energy used, as detailed in Table 2. This was done to ensure that all electron paths were entirely within the material (this was determined by trial and error in early test runs). The chemical composition of the solvent was $\mathrm{C}_{32} \mathrm{H}_{134} \mathrm{O}_{52} \mathrm{NaCl}$, with a density of $1.184 \mathrm{~g} \mathrm{~cm}^{-3}$; the chemical composition of the crystal was $\mathrm{C}_{766} \mathrm{H}_{1603} \mathrm{~N}_{193} \mathrm{O}_{435} \mathrm{~S}_{10} \mathrm{NaCl}$, with a density of $1.259 \mathrm{~g} \mathrm{~cm}^{-3}$.

\subsubsection{Set Up Microscope}

CASINO permits simulation of a range of energies in a single execution of the programme, provided the step size is constant. As we had a non-constant step size, we simulated a single energy per execution (see Table 2). For each execution, we simulated 100,000 electrons with a beam radius of $1 \mathrm{~nm}$. We intended the electron beam to represent a point-source origin of PEs in the material, and with a maximum resolution of $20 \mathrm{~nm}$ in the later steps of our simulation, this was considered a suitably small beam to approximate a point.

Table 2. CASINO input parameters. The thickness of the sample was adjusted to ensure electron pathways did not exit the sample. The electron energy was slightly different for the solvent and the crystal as differences in chemical composition resulted in a slightly different average ionisation energy.

\begin{tabular}{rrrrrrr}
\hline X-ray Energy, $\boldsymbol{E}_{\boldsymbol{X}},[\mathrm{keV}]:$ & $\mathbf{9}$ & $\mathbf{1 2}$ & $\mathbf{1 5}$ & $\mathbf{2 0}$ & $\mathbf{2 5}$ & \multicolumn{1}{c}{$\mathbf{3 0}$} \\
\hline Photoelectron Energy, $\boldsymbol{E}_{\boldsymbol{P E}},($ Solvent) $[\mathrm{keV}]:$ & 7.89 & 10.85 & 13.83 & 18.80 & 23.77 & 28.75 \\
Photoelectron Energy, $\boldsymbol{E}_{\boldsymbol{P E}},($ Crystal) $[\mathrm{keV}]:$ & 7.81 & 10.77 & 13.73 & 18.69 & 23.67 & 28.64 \\
\hline Sample Thickness $[\mathbf{n m}]:$ & 2000 & 4000 & 5000 & 10,000 & 15,000 & 20,000 \\
\hline
\end{tabular}

\subsubsection{Select Distributions}

We enabled "Distribution of Maximum depth of electrons" with 1000 divisions, and "Division of energy by position" with 200 divisions in each axis $(x, y$, and $z)$. All other distributions were disabled as their output was not relevant to the remainder of our simulation. 


\subsection{Model Pt SrC}

Energy deposition by position data (E Dep Map in Figure 6) were exported from CASINO and subsequently processed using MATLAB. The Monte Carlo simulations of electron trajectory were interpreted in the follow way: the simulated trajectories represented the statistical distribution of paths followed by electrons produced at a particular point in space (in CASINO, the meeting of the electron beam with the sample) and moving initially in a particular direction (in CASINO, straight down, along the $z$-axis). The data output by CASINO therefore required post-processing to account for the fact that photoelectrons may be produced at any point within the beam footprint (with the likelihood of emission at a given point being a function of the beam intensity at that point) and that any photoelectrons produced at a given point would have a probability of being emitted in a given direction that was dependent upon the polarisation of the X-ray beam.That is, for a linearly polarised beam, the probability of emission in a given direction would go as $\cos ^{2}(\theta)$, where $\theta$ is the angle made to the polarisation vector. The former requirement is addressed below in Sections 4.4 and 4.5. The MATLAB script used to model the latter of these requirements proceeded in the following basic sequence:

(1) Read CASINO energy-by-position data into an array, centred on the emission location of the PE.

(2) In steps of $1^{\circ}$, rotate the array, scale it by $\cos ^{2}(\theta)$, where $\theta$ was the angle made to the $x$-axis (as we were simulating a horizontal linear polarised X-ray beam), and sum the results for $\theta=[0,360)$.

(3) Scale the array to sum to $E_{e^{-}}=E_{X}-E_{K}$, where $E_{e^{-}}$was the average energy of a PE produced in the sample, $E_{X}$ was the X-ray beam energy, and $E_{K}$ was the average $K$-shell energy in the sample weighted by chemical composition and elemental cross-section. That is, the array ( $\mathrm{Pt} \mathrm{SrC}$ E Map in Figure 6) represents the average distribution of energy (about a given point) due to a single photoionisation event.

\subsection{Model Beam}

The beam footprint was generated as a 2D Gaussian array which was scaled to sum to the total beam flux of $1 \times 10^{10}$ photons s$^{-1}$. This array and the relevant interaction cross-sections (see Section 4.1) were then used to generate arrays whose elements were the rate of PE emission, Compton scattering, and coherent scattering (collectively Interaction Rates in Figure 6).

\subsection{Model Energy Deposition}

An array representing the energy deposited in the entire beam footprint (2D Dose Map in Figure 6) was produced by summing arrays representing the footprint due to photoelectric absorption and that due to Compton scattering. Two versions of the former were used: one modelled energy deposition due to photoelectric absorption in the absence of PE escape (the control case), which was obtained by multiplying the PE emission rate array by the average PE energy; and the other accounted for PE escape and was obtained by convolving the PE emission rate array with Pt Src E Map. The array representing energy deposition due to Compton scattering was modelled by multiplying the Compton scattering rate array by the average Compton recoil energy, which we took to be half the maximum recoil energy (see note below in Section 4.9). The resulting 2D array represented a slice through the beam orthogonal to its axis; energy deposition was assumed to be isotropic in the $z$-direction (parallel to the X-ray beam). Energy values were converted to dose based on the density of the crystal and the volume of a single voxel. One dose map was produced per set of beam/sample configurations. Finally, the array was cropped to the size of the crystal, assumed to be centred in the beam.

\subsection{RADDOSE-3D}

RADDOSE-3D was used as a comparative tool to produce 3D dose maps with sample- and beam-parameters matched, as closely as possible, to those used for the 2D dose maps. RADDOSE-3D is in continuous development, with updates being made available to users via their GitHub repository (https://github.com/GarmanGroup/RADDOSE-3D). We downloaded what was then the current 
stable version of RADDOSE-3D [9] on 22 June 2017, and used this version and the output it produced in all work presented in this study. The version we used did not include either Compton scattering or PE escape; recently, a new version has been made available that includes both these effects.

\subsection{Model Spot Intensity}

The damage state of the crystal was represented by an array whose elements were values between 0 and 1 , with 1 representing a completely undamaged region of the crystal, which would contribute strongly to the diffracted intensity, and 0 representing a completely disordered region. Each element of this array was linked to the element of the dose rate array with the corresponding row- and column-indices. The global dose (when accounting for both PE escape and Compton scattering) was used to determine the time required for the crystal to absorb a total dose of $60 \mathrm{MGy}$ and this time was split into 300 equal time steps. At each time step, Equation (1) was used to calculate the values of the damage array, with $I_{0}$ normalised to 1 . $H$ was Howell's criterion $\left(10 \mathrm{MGy} \AA^{-1}\right)$, and $d$ was $3 \AA$, the resolution of the Bragg spots we were simulating. For example, if the element of the dose rate array with indices $x_{1}, y_{1}$ held the value $0.3 \mathrm{MGy} \mathrm{s}^{-1}$, at time step $t=30 \mathrm{~s}$ the element of the damage array with indices $x_{1}, y_{1}$ would be set to

$$
\exp \left(-\ln (2) \frac{0.3 \mathrm{MGy} \mathrm{s}^{-1} \cdot 30 \mathrm{~s}}{10{\mathrm{MGy} \AA^{-1} \cdot 3 \AA}_{\AA}}\right)=0.8123 .
$$

At each time step, the elements of the damage array were summed and the total divided by the summed value of the array at time $t=0 \mathrm{~s}$. For each combination of crystal size, beam FWHM, and beam energy, this spot-fading model was run using three dose maps: the 2D dose maps generated as described in Section 4.5, one accounted for both PE escape and Compton scattering, one did not include PE escape but did include Compton scattering, and the third set of dose maps was produced using RADDOSE-3D (the script was altered slightly to account for the RADDOSE-3D maps being 3D rather than 2D).

At steps of $15 \mathrm{MGy}$, a qualitative indication of which parts of the crystal were contributing most to diffracted intensity was obtained by taking a point-wise product of the coherent scatter and damage arrays to produce a spatially-resolved map of diffraction-contribution. Reference to the coherent scatter array accounted for the fact that, even if a region of the crystal were largely undamaged, if there was minimal flux through that region, it would not contribute strongly to the diffracted intensity.

\subsection{Calculations of Diffraction Efficiency}

We calculated diffraction efficiency by two methods: (i) using cross-section data from the NIST XCOM database [25]; and (ii) comparing the total coherent scatter to the global dose for each of our simulated data-sets. For calculations based on cross-sections for coherent scatter, $\sigma_{C}$, incoherent scatter, $\sigma_{I}$, and photoelectric absorption, $\sigma_{P}$, the diffraction efficiency was calculated as

$$
D E(E)=\frac{\sigma_{C}}{\sigma_{I}+\sigma_{P}} .
$$

$D E(12 \mathrm{keV})$ was our reference value and we expressed all other values as a ratio $D E(E) / D E(12 \mathrm{keV})$. For each of our simulated data sets, we calculated the diffraction efficiency (per unit volume) as

$$
D E(E)=\frac{N_{C}}{\sigma_{X}^{3} D_{G}},
$$

where $N_{C}$ is the number of elastically scattered photons, $\sigma_{X}$ is the side-length of the crystal, and $D_{G}$ is the global dose in MGy. The reference value used was the diffraction efficiency of a $1 \mu \mathrm{m}$ crystal in a $1 \mu \mathrm{m}$ beam at $12 \mathrm{keV}$, as calculated using the RADDOSE-3D dose maps, and all other values were expressed as a ratio to this reference. 


\subsection{Simplifications and Assumptions in Our Model}

We simulated the loss of integrated intensity of a single $3 \AA$ Bragg spot (or, equivalently, all $3 \AA$ spots in the diffraction pattern). Three angstroms was chosen as a reasonable minimal desirable resolution, though ultimately the choice is arbitrary. While it is noted in the literature that loss of integrated intensity is commonly used as a metric for radiation damage and that this may be measured in a full-data set, a repeated wedge, or single peak [13], it is not clear that these observations are necessarily equivalent. It is expected that simulating a single resolution is sufficient to provide a qualitative measure of the effect of including or excluding PE escape in simulations of radiation damage, which is the focus of this work. In theory, the simulation could be expanded to account for the fading of all spots in a diffraction pattern, between given minimum and maximum resolution, though this would significantly increase execution time.

The RADDOSE-3D documentation [28] for the version we used in this study stated that it was not accurate at higher beam energies $(>20 \mathrm{keV})$, advising users to instead use RADDOSE v2 via the online web server (http://www.raddo.se/legacy/). The cause and nature of this inaccuracy were not given. RADDOSE v2 does not produce spatially-resolved dose maps and provides the maximum dose for the case of a Gaussian beam, rather than the average dose across the beam profile [11]. The former point precluded us from using it in our spot-fading models; we used RADDOSE-3D at all energies. To determine what effect this stated lack of accuracy may have had on the legitimacy of comparison between our simulations and RADDOSE-3D, we compared the dose predictions of RADDOSE v2 with the maximum dose predicted by RADDOSE-3D at energies above $20 \mathrm{keV}$, for all crystal and beam sizes used in our simulations. In all cases, the RADDOSE v2 dose was higher than the RADDOSE-3D maximum dose by approximately 13\%. Accordingly, we would expect results obtained with RADDOSE-3D to underestimate damage rates at higher energies, compared to RADDOSE v2.

In post-processing CASINO energy-deposition data to generate a 2D map of energy deposition due to a single photoionisation event, we took the intersection of the beam and sample surface (in CASINO) to be the origin of the photoionisation. A more precise model would take this point as the first collision in which the photoelectron was involved. That is, this point should be one IMFP away from the origin of photoionisation, potentially leaving a small area of reduced damage at the centre of the pattern. At the resolutions we used in our simulations, this is unlikely to cause significant error as the energy deposition will be displaced by only $2-5$ pixels, depending on beam energy.

We made the simplifying assumption that the average Compton recoil energy was half the maxim recoil energy. Assuming a uniform distribution of Compton scattering angles (between $0^{\circ}$ and $180^{\circ}$ ), an average taken over recoil energies calculated in $0.5^{\circ}$ steps differs from this value by only $1-3 \%$ in the energy range we simulated, so this simplification has minimal impact on the outcome, with our simulations slightly under-predicting the impact of Compton scattering on dose.

\section{Conclusions}

We performed spatially- and temporally-resolved simulations of radiation damage to protein crystals during micro-crystallography experiments that account for the escape of photoelectrons from the diffraction volume. Our simulations explored damage rates for $1 \mu \mathrm{m}, 1.5 \mu \mathrm{m}, 2.0 \mu \mathrm{m}$ and $5 \mu \mathrm{m}$ crystals in beams with energy from $9 \mathrm{keV}$ to $30 \mathrm{keV}$ and beam size that was either matched to or larger than the crystal size. By accounting for the escape of photoelectrons from the diffraction volume, our simulations predict longer lifetimes (when compared to control simulations and simulations performed using a version of RADDOSE-3D that did not account for PE escape), and increased diffraction efficiency (by a factor of between 7 and 30), for smaller crystals $(<5 \mu \mathrm{m})$, provided the beam focus is not much larger than the crystal itself, and when using higher beam energy $(\geqslant 20 \mathrm{keV})$. These results suggest significant benefit may be realised by conducting MX experiments on microcrystals at around $20 \mathrm{keV}$ or higher. A systematic experimental exploration of radiation damage under the range of parameters we have simulated is now needed to validate and refine our models. 
Author Contributions: Conceptualization, C.D. and B.A.; Formal analysis, H.M.; Methodology, H.M.; Project administration, B.A.; Software, H.M.; Supervision, C.D. and B.A.; Writing-original draft, H.M.; Writing一review \& editing, C.D. and B.A.

Funding: This work was supported by the Australian Research Council Centre of Excellence in Advanced Molecular Imaging (CE140100011) (http:/ / www.imagingcoe.org/).

Conflicts of Interest: The authors declare no conflict of interest.

\section{Abbreviations}

The following abbreviations are used in this manuscript:

$\begin{array}{ll}\text { BCDI } & \text { Bragg coherent diffractive imaging } \\ \text { FWHM } & \text { full-width at half-maximum } \\ \text { IMFP } & \text { inelastic mean free path } \\ \text { MC } & \text { Monte Carlo } \\ \text { MX } & \text { macromolecular crystallography } \\ \text { PE } & \text { photoelectron } \\ \text { RSM } & \text { reciprocal space map(ping) }\end{array}$

\section{Appendix A. Diffraction Efficiency for Simulations with No Photoelectron Escape}

Diffraction efficiency of all individual simulated data sets that did not incorporate PE escape are shown in Tables A1 and A2, with averages and standard deviation.

Table A1. Diffraction efficiency, relative to the $12 \mathrm{keV}$ value for RADDOSE-3D simulations, of all simulated data sets based on our control dose maps that included Compton scattering but excluded PE escape. The data show minimal variation with beam and crystal size.

\begin{tabular}{rcccccc}
\hline Energy $[\mathrm{keV}]:$ & $\mathbf{9}$ & $\mathbf{1 2}$ & $\mathbf{1 5}$ & $\mathbf{2 0}$ & $\mathbf{2 5}$ & $\mathbf{3 0}$ \\
\hline$\sigma_{X}=1 \mu \mathrm{m}, \sigma_{B}=1 \mu \mathrm{m}:$ & 0.81 & 0.99 & 1.15 & 1.37 & 1.51 & 1.63 \\
$\sigma_{X}=1 \mu \mathrm{m}, \sigma_{B}=1.5 \mu \mathrm{m}:$ & 0.81 & 0.99 & 1.15 & 1.37 & 1.51 & 1.63 \\
$\sigma_{X}=1 \mu \mathrm{m}, \sigma_{B}=2 \mu \mathrm{m}:$ & 0.81 & 0.99 & 1.15 & 1.37 & 1.51 & 1.63 \\
$\sigma_{X}=1 \mu \mathrm{m}, \sigma_{B}=5 \mu \mathrm{m}:$ & 0.81 & 0.99 & 1.15 & 1.37 & 1.51 & 1.63 \\
$\sigma_{X}=1.5 \mu \mathrm{m}, \sigma_{B}=1.5 \mu \mathrm{m}:$ & 0.81 & 0.99 & 1.15 & 1.40 & 1.51 & 1.56 \\
$\sigma_{X}=2 \mu \mathrm{m}, \sigma_{B}=2 \mu \mathrm{m}:$ & 0.81 & 0.99 & 1.15 & 1.37 & 1.51 & 1.53 \\
$\sigma_{X}=5 \mu \mathrm{m}, \sigma_{B}=5 \mu \mathrm{m}:$ & 0.81 & 0.99 & 1.15 & 1.37 & 1.51 & 1.55 \\
\hline Average: & 0.81 & 0.99 & 1.15 & 1.37 & 1.51 & 1.59 \\
Standard Deviation (\%): & $(0.00 \%)$ & $(0.00 \%)$ & $(0.00 \%)$ & $(1.01 \%)$ & $(0.00 \%)$ & $(2.67 \%)$ \\
\hline
\end{tabular}

Table A2. Diffraction efficiency, relative to $12 \mathrm{keV}$, of all simulated data sets based on RADDOSE-3D [9] dose maps that did not account for either Compton scattering or PE escape. The data show minimal variation with beam and crystal size.

\begin{tabular}{rcccccc}
\hline Energy [keV]: & $\mathbf{9}$ & $\mathbf{1 2}$ & $\mathbf{1 5}$ & $\mathbf{2 0}$ & $\mathbf{2 5}$ & $\mathbf{3 0}$ \\
\hline$\sigma_{X}=1 \mu \mathrm{m}, \sigma_{B}=1 \mu \mathrm{m}:$ & 0.79 & 1.00 & 1.19 & 1.46 & 1.69 & 1.88 \\
$\sigma_{X}=1 \mu \mathrm{m}, \sigma_{B}=1.5 \mu \mathrm{m}:$ & 0.79 & 1.00 & 1.19 & 1.46 & 1.70 & 1.87 \\
$\sigma_{X}=1 \mu \mathrm{m}, \sigma_{B}=2 \mu \mathrm{m}:$ & 0.79 & 1.00 & 1.19 & 1.46 & 1.70 & 1.88 \\
$\sigma_{X}=1 \mu \mathrm{m}, \sigma_{B}=5 \mu \mathrm{m}:$ & 0.79 & 1.00 & 1.19 & 1.46 & 1.70 & 1.88 \\
$\sigma_{X}=1.5 \mu \mathrm{m}, \sigma_{B}=1.5 \mu \mathrm{m}:$ & 0.79 & 1.00 & 1.19 & 1.48 & 1.69 & 2.03 \\
$\sigma_{X}=2 \mu \mathrm{m}, \sigma_{B}=2 \mu \mathrm{m}:$ & 0.79 & 1.00 & 1.20 & 1.47 & 1.69 & 1.94 \\
$\sigma_{X}=5 \mu \mathrm{m}, \sigma_{B}=5 \mu \mathrm{m}:$ & 0.79 & 1.00 & 1.19 & 1.47 & 1.70 & 1.90 \\
\hline Average: & 0.79 & 1.00 & 1.19 & 1.47 & 1.69 & 1.91 \\
Standard Deviation (\%): & $(0.05 \%)$ & $(0.13 \%)$ & $(0.12 \%)$ & $(0.5 \%)$ & $(0.18 \%)$ & $(3.06 \%)$ \\
\hline
\end{tabular}

\section{Appendix B. Global Dose Comparisons}

The RADDOSE-3D documentation (for the version we used in this study) states that dose predictions are known to be inaccurate above $20 \mathrm{keV}$ and recommends the use of RADDOSE v2, 
which is not spatially-resolved, in this energy regime. As we required spatial-resolution for our comparisons, we had to use RADDOSE-3D at all energies. To determine what effect this would have on our comparisons, we repeated global dose predictions for energies above $20 \mathrm{keV}$ with RADDOSE v2 as well as RADDOSE-3D. RADDOSE v2 gives a maximum dose when using Gaussian beams, rather than an average dose. We compared it to the maximum dose as calculated using RADDOSE-3D. As Table A3 shows, RADDOSE-3D predictions are lower than RADDOSe v2 predictions.

Table A3. Global dose, as calculated by our simulations, RADDOSE-3D [9], and (for beam energies $\geqslant 20 \mathrm{keV}$ ) RADDOSE $\mathrm{v} 2$, for a $1 \mu \mathrm{m}$ crystal in a $1 \mu \mathrm{m}$ (FWHM) beam with flux of $1 \times 10^{10}$ photons s$^{-1}$ and energy ranging from $9 \mathrm{keV}$ to $30 \mathrm{keV}$. The total dose calculated by RADDOSE v2 for Gaussian beams is the maximum dose, not the average, hence, we compared it to the maximum dose reported by RADDOSE-3D.

\begin{tabular}{ccccc}
\hline $\begin{array}{c}\text { Energy } \\
\text { [keV] }\end{array}$ & $\begin{array}{c}\text { Our Simulation } \\
\text { (Average) }\end{array}$ & $\begin{array}{c}\text { Dose [MGy] } \\
\text { (Average) }\end{array}$ & $\begin{array}{c}\text { RADDOSE-3D } \\
\text { (Maximum) }\end{array}$ & $\begin{array}{c}\text { RADDOSE v2 } \\
\text { (Maximum) }\end{array}$ \\
\hline 9 & 2.70 & 5.26 & 8.02 & - \\
12 & 0.78 & 2.83 & 4.32 & - \\
15 & 0.29 & 1.74 & 2.65 & - \\
20 & 0.09 & 0.92 & 1.40 & 1.59 \\
25 & 0.04 & 0.56 & 0.85 & 0.97 \\
30 & 0.03 & 0.37 & 0.57 & 0.64 \\
\hline
\end{tabular}

\section{References}

1. Hettel, R. DLSR design and plans: An international overview. J. Synchrotron Radiat. 2014, 21, $843-855$. [CrossRef] [PubMed]

2. Schroer, C.G.; Falkenberg, G. Hard X-ray nanofocusing at low-emittance synchrotron radiation sources. J. Synchrotron Radiat. 2014, 21, 996-1005. [CrossRef] [PubMed]

3. Garman, E.F.; Weik, M. Radiation Damage in Macromolecular Crystallography. Synchrotron Radiat. News 2015, 28, 15-19. [CrossRef]

4. Garman, E.F.; Weik, M. Radiation damage to macromolecules: Kill or cure? J. Synchrotron Radiat. 2015, 22, 195-200. [CrossRef] [PubMed]

5. Garman, E.F.; Weik, M. Radiation damage to biological macromolecules: Some answers and more questions. J. Synchrotron Radiat. 2013, 20, 1-6. [CrossRef] [PubMed]

6. Garman, E.F. Radiation damage in macromolecular crystallography: What is it and why should we care? Acta Cryst. D 2010, 66, 339-351. [CrossRef] [PubMed]

7. Holton, J.M. A beginner's guide to radiation damage. J. Synchrotron Radiat. 2009, 16, 133-142. [CrossRef] [PubMed]

8. Howells, M.R.; Beetz, T.; Chapman, H.N.; Cui, C.; Jacobsen, C.J.; Kirz, J.; Lima, E.; Marchesini, S.; Miao, H.; Sayre, D.; et al. An assessment of the resolution limitation due to radiation-damage in X-ray diffraction microscopy. J. Electron Spectrosc. Relat. Phenom. 2009, 170, 4-12. [CrossRef] [PubMed]

9. Zeldin, O.B.; Gerstel, M.; Garman, E.F. RADDOSE-3D: Time- and space-resolved modelling of dose in macromolecular crystallography. J. Appl. Crystallogr. 2013, 46, 1225-1230. [CrossRef]

10. Paithankar, K.S.; Garman, E.F. Know your dose: RADDOSE. Acta Cryst. D 2010, 66, 381-388. [CrossRef] [PubMed]

11. Paithankar, K.S.; Owen, R.L.; Garman, E.F. Absorbed dose calculations for macromolecular crystals: Improvements to RADDOSE. J. Synchrotron Radiat. 2009, 16, 152-162. [CrossRef] [PubMed]

12. Murray, J.W.; Garman, E.F.; Ravelli, R.B.G. X-ray absorption by macromolecular crystals: The effects of wavelength and crystal composition on absorbed dose. J. Appl. Crystallogr. 2004, 37, 513-522. [CrossRef]

13. Garman, E.F.; Weik, M. X-ray radiation damage to biological macromolecules: Further insights. J. Synchrotron Radiat. 2017, 24, 1-6. [CrossRef] [PubMed] 
14. Coughlan, H.D.; Darmanin, C.; Kirkwood, H.J.; Phillips, N.W.; Hoxley, D.; Clark, J.N.; Vine, D.J.; Hofmann, F.; Harder, R.J.; Maxey, E.; et al. Bragg coherent diffraction imaging and metrics for radiation damage in protein micro-crystallography. J. Synchrotron Radiat. 2017, 24, 83-94. [CrossRef] [PubMed]

15. Coughlan, H.D.; Darmanin, C.; Phillips, N.W.; Hofmann, F.; Clark, J.N.; Harder, R.J.; Vine, D.J.; Abbey, B. Radiation damage in a micron-sized protein crystal studied via reciprocal space mapping and coherent diffractive imaging. Struct. Dyn. 2015, 2, 041704. [CrossRef] [PubMed]

16. Finfrock, Y.Z.; Stern, E.A.; Alkire, R.W.; Kas, J.J.; Evans-Lutterodt, K.; Stein, A.; Duke, N.; Lazarski, K.; Joachimiak, A. Mitigation of X-ray damage in macromolecular crystallography by submicrometre line focusing. Acta Cryst. D 2013, 69, 1463-1469. [CrossRef] [PubMed]

17. Sanishvili, R.; Yoder, D.W.; Pothineni, S.B.; Rosenbaum, G.; Xu, S.; Vogt, S.; Stepanov, S.; Makarov, O.A.; Corcoran, S.; Benn, R.; et al. Radiation damage in protein crystals is reduced with a micron-sized X-ray beam. Proc. Natl. Acad. Sci. USA 2011, 108, 6127-6132. [CrossRef] [PubMed]

18. Finfrock, Y.Z.; Stern, E.A.; Yacoby, Y.; Alkire, R.W.; Evans-Lutterodt, K.; Stein, A.; Isakovic, A.F.; Kas, J.J.; Joachimiak, A. Spatial dependence and mitigation of radiation damage by a line-focus mini-beam. Acta Cryst. D 2010, 66, 1287-1294. [CrossRef] [PubMed]

19. Bury, C.S.; Brooks-Bartlett, J.C.; Walsh, S.P.; Garman, E.F. Estimate your dose: RADDOSE-3D. Protein Sci. 2018, 27, 217-228. [CrossRef] [PubMed]

20. Cowan, J.A.; Nave, C. The optimum conditions to collect X-ray data from very small samples. J. Synchrotron Radiat. 2008, 15, 458-462. [CrossRef] [PubMed]

21. Nave, C.; Hill, M.A. Will reduced radiation damage occur with very small crystals? J. Synchrotron Radiat. 2005, 12, 299-303. [CrossRef] [PubMed]

22. Stern, E.A.; Yacoby, Y.; Seidler, G.T.; Nagle, K.P.; Prange, M.P.; Sorini, A.P.; Rehr, J.J.; Joachimiak, A. Reducing radiation damage in macromolecular crystals at synchrotron sources. Acta Cryst. D 2009, 65, 366-374. [CrossRef] [PubMed]

23. Drouin, D.; Couture, A.R.; Joly, D.; Tastet, X.; Aimez, V.; Gauvin, R. CASINO V2.42-A Fast and Easy-to-use Modeling Tool fro Scanning Electron Microscopy and Microanalysis Users. Scanning 2007, 29, 92-101. [CrossRef] [PubMed]

24. Drouin, D.; Hovington, P.; Gauvin, R. CASINO: A New Monte Carlo Code in C Language for Electron Beam Interaction-Part II: Tabulated Values of the Mott Cross Section. Scanning 1997, 19, 20-28. [CrossRef]

25. Berger, M.; Hubbell, J.; Seltzer, S.; Chang, J.; Coursey, J.; Sukumar, R.; Zucker, D.; Olsen, K. XCOM: Photon Cross Sections Database. Available online: http://physics.nist.gov/xcom (accessed on 22 September 2017).

26. Warkentin, M.A.; Atakisi, H.; Hopkins, J.B.; Walko, D.; Thorne, R.E. Lifetimes and spatio-temporal response of protein crystals in intense X-ray microbeams. IUCrJ 2017, 4, 785-794 [CrossRef] [PubMed]

27. Holton, J.M.; Frankel, K.A. The minimum crystal size needed for a complete diffraction data set. Acta Cryst. 2010, D66, 393-408. [CrossRef] [PubMed]

28. RADDOSE-3D Readme File. Available online: https://github.com/GarmanGroup/RADDOSE-3D/blob/ master/README.md (accessed on 21 June 2018).

(C) 2018 by the authors. Licensee MDPI, Basel, Switzerland. This article is an open access article distributed under the terms and conditions of the Creative Commons Attribution (CC BY) license (http://creativecommons.org/licenses/by/4.0/). 